\title{
Microwave-assisted tunneling and interference effects in superconducting junctions under fast driving signals
}

\author{
Piotr Kot, ${ }^{1}$ Robert Drost,,${ }^{1, *}$ Maximilian Uhl,,${ }^{1}$ Joachim Ankerhold, ${ }^{2}$ Juan Carlos Cuevas $\odot,{ }^{3}$ and Christian R. Ast $\oplus^{1}$ \\ ${ }^{1}$ Max-Planck-Institut für Festkörperforschung, Heisenbergstraße 1, 70569 Stuttgart, Germany \\ ${ }^{2}$ Institut für Komplexe Quantensysteme and IQST, Universität Ulm, Albert-Einstein-Allee 11, 89069 Ulm, Germany \\ ${ }^{3}$ Departamento de Física Teórica de la Materia Condensada and Condensed Matter Physics Center (IFIMAC), Universidad Autónoma de \\ Madrid, 28049 Madrid, Spain
}

(Received 23 December 2019; revised manuscript received 11 March 2020; accepted 13 March 2020; published 16 April 2020)

\begin{abstract}
As scanning tunneling microscopy is pushed towards fast local dynamics, a quantitative understanding of tunnel junctions under the influence of a fast ac driving signal is required, especially at the ultralow temperatures relevant to spin dynamics and correlated electron states. We subject a superconductor-insulator-superconductor junction to a microwave signal from an antenna mounted in situ and examine the dc response of the contact to this driving signal. Quasiparticle tunneling and the Josephson effect can be interpreted in the framework of Tien-Gordon theory. The situation is more complex when it comes to higher-order effects such as multiple Andreev reflections. Microwave-assisted tunneling unravels these complex processes, providing deeper insights into tunneling than are available in a pure dc measurement.
\end{abstract}

DOI: 10.1103/PhysRevB.101.134507

\section{INTRODUCTION}

With its combination of supreme spatial resolution and spectroscopic imaging, the scanning tunneling microscope (STM) is a workhorse of mesoscopic and nanoscale physics [1]. The technique remains plagued, however, by the inherently low bandwidth of the transimpedance amplifiers required to measure the small tunnel current. Even so, there is a growing desire to exploit the unique capabilities of the STM to study nanoscale objects on their own time scales. With the integration of high-frequency excitation methods, the development of pump-probe schemes, and the advent of electron spin resonance STM in recent years, this goal appears to be in reach [2-13]. A fast driving signal is generally viewed as a means to excite fundamental modes within the sample and study their dynamics. The effect of the ac signal on the dc conductance is much less well explored. In this context, a quantitative understanding of the response of the tunnel junction itself to a fast driving signal is required, especially at the ultralow temperatures relevant to spin dynamics and correlated electron states.

Previous theoretical and experimental investigations into this direction have focused on tunneling between superconductors where conductance spectra are dominated by sharp

\footnotetext{
${ }^{*}$ Corresponding author: r.drost @ fkf.mpg.de
}

Published by the American Physical Society under the terms of the Creative Commons Attribution 4.0 International license. Further distribution of this work must maintain attribution to the author(s) and the published article's title, journal citation, and DOI. Open access publication funded by the Max Planck Society. peaks, which make the effect of microwave radiation easy to discern [14-18]. This situation is especially interesting as superconductor-insulator-superconductor (SIS) junctions support multiple Andreev reflections (MARs), permitting us to study the interaction of microwave radiation with higher-order tunneling processes. On the theoretical level, the problem is treated semiclassically with the microwave signal leading to a time-dependent modulation of the bias voltage dropping across the junction. Quasiparticles and Cooper pairs thus show a similar reaction to the incident radiation despite their stark differences in physical origin. It has been suggested that microwave radiation couples to the tunnel current through the total charge transferred between the electrodes in any given process [18].

Most data up to date has been analyzed in the framework of the Tien-Gordon model, which treats tunneling and microwave interaction as independent processes $[15,16]$. More advanced theories suggest that SIS tunneling under microwave irradiation must be understood through MARs while accounting for microwave interactions at every step of these higher-order processes [19]. In this MAR model, the microwave signal is modeled as a time-dependent phase difference between tip and sample electrodes and MARs arise naturally through higher-order terms. Interference between particles traveling back and forth within the barrier are predicted to yield a very different result than the Tien-Gordon approach. Here, we report measurements of quasiparticle, Cooper pair, and MAR tunneling in an SIS junction under microwave irradiation. These experimental findings are compared with predictions from the Tien-Gordon and the microwave-assisted MAR models, respectively. Only the latter one is found to be capable of correctly modeling the impact of microwave driving on the charge transfer process. 

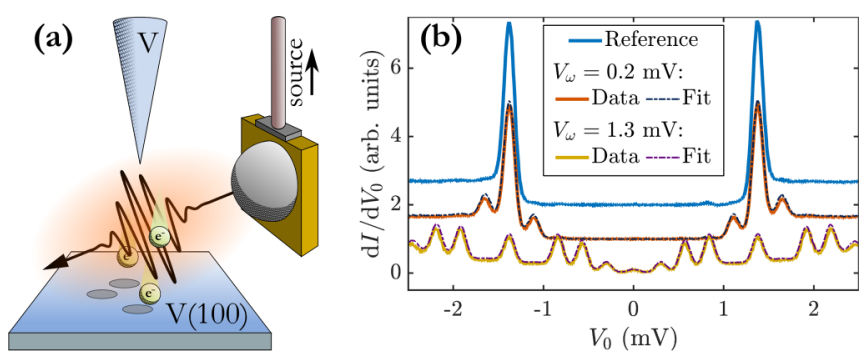

FIG. 1. Microwave-assisted tunneling in an SIS junction: (a) Sketch of the experimental setup. A commercial STM is fitted with a radio frequency antenna delivering radiation to the junction. (b) Conductance spectrum in the tunneling regime without microwave signal (light blue) and under microwave radiation at $65 \mathrm{GHz}$ and two different power settings (orange with $V_{\omega}=0.2 \mathrm{mV}$ and yellow with $V_{\omega}=1.3 \mathrm{mV}$ ) with corresponding fits according to Eq. (1) in orange and purple, respectively. $G_{N} \approx 8.4 \times 10^{-3} G_{0}$ for all spectra.

\section{EXPERIMENTAL}

We study SIS tunneling between a V(100) surface and a vanadium tip in a commercial STM system (Unisoku USM1300) operating at $300 \mathrm{mK}$ base temperature and fitted with a custom-built antenna assembly capable of delivering a microwave signal between $60-90 \mathrm{GHz}$ to the junction, see Fig. 1(a). The V(100) sample is prepared by repeated bombardment with $\mathrm{Ar}^{+}$ions at $1 \mathrm{keV}$ and annealing at $\approx 650^{\circ} \mathrm{C}$, resulting in the well-known $(5 \times 1)$ oxygen reconstruction $[20,21]$. The tip is cut from vanadium wire and cleaned by bombardment with $\mathrm{Ar}^{+}$ions before being transferred into the STM and further prepared by controlled indentation into the $\mathrm{V}(100)$ surface until a clean SIS signature is observed.

\section{MEASUREMENT AND DISCUSSION}

We begin our investigation in the tunneling regime at large tip-sample distances where the normal state conductance $G_{N}$ is only a small fraction of the quantum of conductance $G_{0}=$ $2 e^{2} / h$, where $e$ is the elementary charge and $h$ the Planck constant. Conductance spectra acquired above the pristine $\mathrm{V}(100)$ surface show behavior typical for SIS junctions. A representative data set is shown in Fig. 1(b) in light blue. The primary features are two sharp coherence peaks separated by twice the sum of the superconducting gap in tip and sample, $\Delta_{\text {tip }}=663 \mu \mathrm{eV}$ and $\Delta_{\text {sample }}=710 \mu \mathrm{eV}$ (see Supplemental Material [22] and Refs. [23-26] therein for details). Such a low-temperature, low-capacitance junction under ac driving has previously been considered by Falci et al., who found the following expression for the time-averaged dc tunnel current, also known as the Tien-Gordon equation [15,16,22]:

$$
I\left(V_{0}, V_{\omega}\right)=\sum_{n=-\infty}^{\infty} J_{n}^{2}\left(\frac{e \mathrm{~V}_{\omega}}{\hbar \omega}\right) I_{0}\left(V_{0}-\frac{n \hbar \omega}{e}\right) .
$$

Here, $V_{0}$ is the dc junction bias, $V_{\omega}$ the amplitude of the ac voltage resulting from the incident microwave, $J_{n}$ are the Bessel functions of the first kind and order $n, e$ is the elementary charge, $\hbar$ the reduced Planck constant, $\omega$ the angular frequency of the microwave signal, and $I_{0}$ is the tunnel current in absence of irradiation. The same relationship follows for the conductance signal $G\left(V_{0}, V_{\omega}\right)=\frac{\mathrm{d}}{\mathrm{dV}_{0}} I\left(V_{0}, V_{\omega}\right)$. Note that though we study a SIS junction in the present case, Eq. (1) will also hold for normal conducting samples.

We thus expect to observe a weighted replication of the original signal at integer multiples of $\hbar \omega$ when subjecting the junction to a microwave signal. This is indeed what is observed in previous experiments [27-29]. Data from a tunnel junction under microwave irradiation is shown in Fig. 1(b) for two different source power settings in dark blue and yellow to highlight the effect of changing $V_{\omega}$. The initial effect of a low-power microwave signal is a diminishing of the coherence peaks at $\pm\left(\Delta_{\text {tip }}+\Delta_{\text {sample }}\right)$, coupled with the appearance of satellite peaks offset by $\pm \hbar \omega$ [see dark blue curve in Fig. 1(b)]. As the power level of the microwave source is increased, higher-order replica of the coherence peaks appear at integer multiples of $\pm \hbar \omega$ [see yellow curve in Fig. 1(b) ]. We use Eq. (1) to fit the experimental data using a reference spectrum, acquired in absence of microwave radiation at the same sample location, and the known value for $\omega$, set at the source module, as input with $V_{\omega}$ as the only free parameter. Fits to the experimental data are shown in orange and purple in Fig. $1(\mathrm{~b})$.

The predicted weighting of the replica by $J_{n}^{2}\left(e \mathrm{~V}_{\omega} / \hbar \omega\right)$ becomes apparent when varying the source power level, and thereby $V_{\omega}$, at fixed frequency. We performed such a measurement, acquiring a series of conductance curves at a frequency of $65 \mathrm{GHz}$ with amplitudes $V_{\omega}$ between 0 and $3.8 \mathrm{mV}$. Each curve is fitted using Eq. (1) to extract $V_{\omega}$. The resulting data set is shown as a stacked contour plot in Fig. 2(a). The coherence peaks fan open as $V_{\omega}$ gradually increases and the spectral intensity of the principal peaks is distributed over a wider energy range. A rich interference pattern emerges in a widening area around zero bias as replica from above and below the Fermi level are woven together. The resulting pattern can be fully understood by the superposition of the coherence peak replica. The agreement between the measured data and the model in Eq. (1), shown in Fig. 2(b), is remarkable.

It is important to note that the energy offset of $\hbar \omega$ between the peak replica should not be interpreted in terms of photon absorption from the microwave field during the tunneling process. Instead, Eq. (1) is the result of the quantum mechanical motion of the electron in a time-dependent classical potential of the form $U(t)=e V_{\omega} \cos (\omega t)$. The inclusion of such a potential will naturally lead to the emergence of the Bessel functions and the resulting eigenenergies will contain components at $E_{0}, E_{0} \pm \hbar \omega, E_{0} \pm 2 \hbar \omega$ and so on [15]. As such, the emergence of the satellite peaks in microwave assisted tunneling are a manifestation of the quantum mechanical nature of electrons in solids. Indeed, the work performed by the classical microwave field on electrons surpasses the photon energy by far, thus placing the experiment in a field-driven regime [22,30].

We extract profiles at constant $V_{0}$, shown in Fig. 2(c), from the data and normalize the resulting curves to the maximum intensity of the coherence peak at $V_{\omega}=0$. The resulting profiles follow their respective low-order Bessel functions almost exactly. Minor discrepancies arise only at low $V_{\omega}$, where the normal conducting background signal has a significant contribution. 

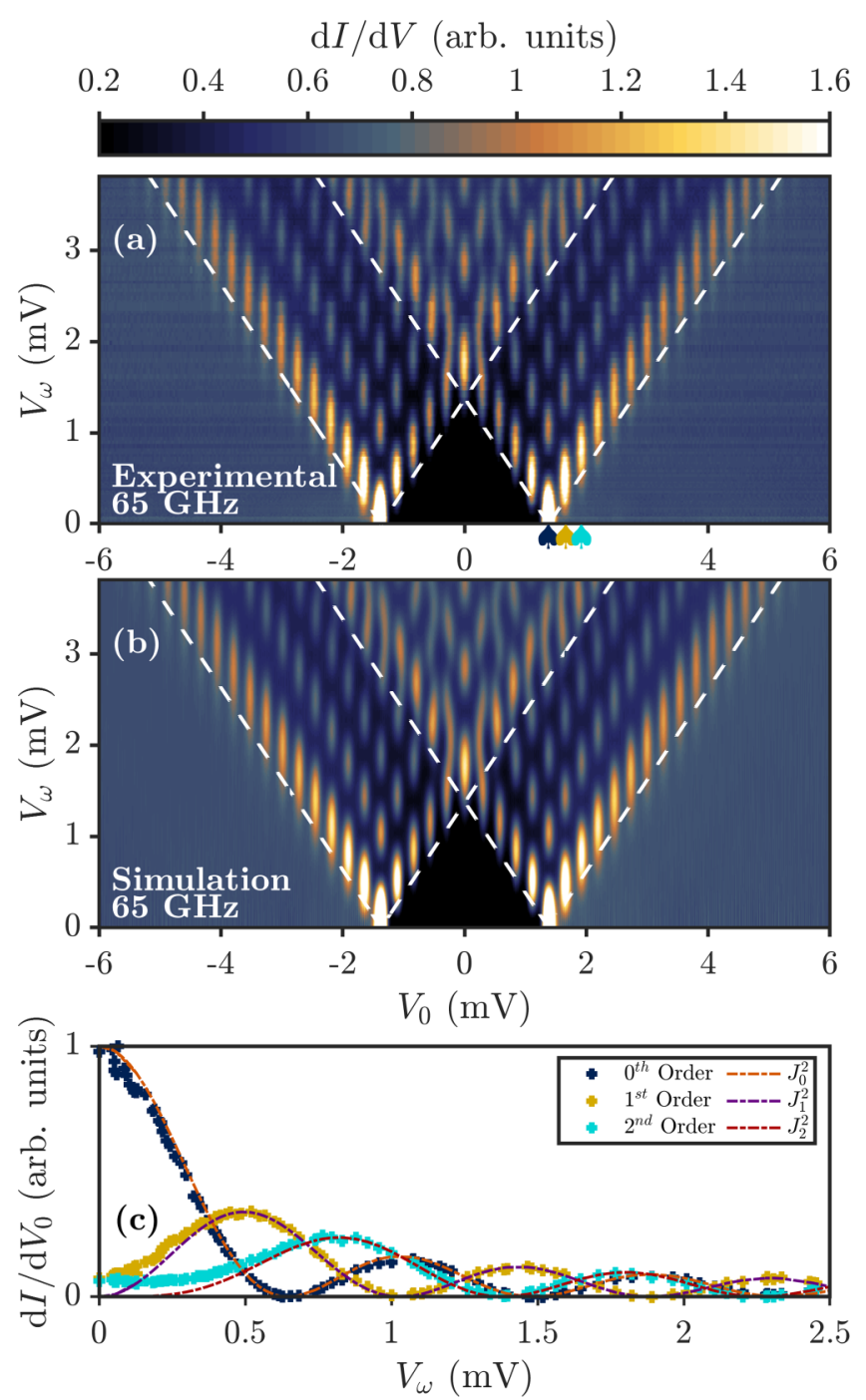

FIG. 2. $V_{\omega}$ dependence of microwave-assisted tunneling: (a) Stacked contour plot of a series of conductance spectra of an SIS junction under irradiation by a $65 \mathrm{GHz}$ signal at varying $V_{\omega}$. Markers on the $x$ axis indicate the locations of the line profiles presented in (c). The white dashed lines demarcate the energy of the coherence peaks $\pm V_{\omega}$. $G_{N}=8.4 \times 10^{-3} G_{0}$. (b) Simulation of the data shown in (a) using Eq. (1). (c) Constant $V_{0}$ profiles of the experimental data in (a) at the principal coherence peak $\left(V_{0}=1.368 \mathrm{mV}\right.$, dark blue) and the first $\left(V_{0}=1.648 \mathrm{mV}\right.$, yellow $)$ and second $\left(V_{0}=1.910 \mathrm{mV}\right.$, cyan) satellites, normalized to the maximum of the 0th-order peak. The dashed-dotted lines are the Bessel functions of zeroth (orange), first (purple), and second (red) order in $\frac{e V_{\omega}}{\hbar \omega}$.

It is interesting to note that Eq. (1) gives an accurate description of the junction even as $e V_{\omega} \gg 2 \Delta_{\text {sample }}$ (approximately $1.52 \mathrm{mV}$ for the present case of vanadium). To push the limits of the model, we examined the behavior of a typical SIS junction when subjected to the highest intensity of microwave radiation possible in our setup at $70.02 \mathrm{GHz}$ where $V_{\omega}$ reaches $13.62 \mathrm{mV}$ and thus $e V_{\omega} \gg 2 \Delta_{\text {sample. The corresponding data }}$ is shown in Fig. 3 (the in-gap peaks in the reference spectrum in Fig. 3 arise from a magnetic impurity state on the tip). Even in these extreme conditions, the experimental data is still well described by the Tien-Gordon Eq. (1). Given that an unperturbed reference spectrum is used in our modeling, this result suggests that the superconductor remains undisturbed by the microwave signal and does not heat up significantly, even under these extreme conditions [22]. Indeed, the superconductor is transparent at energies $\hbar \omega<2 \Delta_{\text {sample }}$ as there are no final states available into which Cooper pairs could be excited [31].

A closer look at the Josephson effect further offers the possibility to study the effect of microwave radiation on tunneling between coherent electron states. Such measurements have previously been performed by Roychowdhury and coworkers, also employing the theoretical description by Falci et al., who gave an expression strikingly similar to the Tien-Gordon equation for the case of the Josephson current in the tunneling regime $[16,18,32]$ :

$$
I\left(V_{0}, V_{\omega}\right)=\sum_{n=-\infty}^{\infty} J_{n}^{2}\left(\frac{2 e \mathrm{~V}_{\omega}}{\hbar \omega}\right) I_{0}\left(V_{0}-\frac{n \hbar \omega}{2 e}\right) .
$$

The only difference to Eq. (1) is the replacement $e \rightarrow 2 e$. Despite their stark difference in physical origin, the QP and the Cooper pair current show the same functional dependencies in their interactions with the microwave radiation. In a second series of conductance spectra, recorded while varying the source power level as for Fig. 2, but at higher contact transmission, shown as a contour plot in Fig. 4 we measured the Josephson effect in the presence of a microwave signal of increasing amplitude. Experimental data is presented in Fig.4(a). The Josephson peak at zero bias is gradually fanning open with increasing $V_{\omega}$. Contributions from MARs and the coherence peaks soon begin to influence the relevant voltage range and restrict the measurement to small $V_{\omega}$. The observed pattern is a result of sequential Cooper pair tunneling in a low-capacitance junction and cannot be understood in terms of Shapiro steps, which require the phase difference across the junction to be a good quantum number [22]. In the tunnel junction of an STM at ultralow temperatures, the low junction capacitance leads to significant phase fluctuations, such that the Josephson effect has to be modeled as a sequential process within the $P(E)$ theory $[16,18,32-36]$. A simulation of the data using Eq. (2) is shown in Fig. 4(b). Also in this case, the peak profiles at constant $V_{0}$ follow the predicted Bessel function dependency with great accuracy, as can be seen in Fig. 4(c).

Given the series of features offset by integer fractions of $\hbar \omega$, it is tempting to think of the tunnel current as being carried by a series of dressed electron states with total charge $q=m e$, where $m=1$ for $\mathrm{QP}$ tunneling, $m=2$ for Cooper pair tunneling and $m \geqslant 2$ for MARs, and each governed by an equation of the form of Eqs. (1) and (2) with the appropriate charge. Indeed, such an interpretation of the microwave radiation coupling to the total charge transferred between tip and sample during a tunnel process has been put forward [18]. This idea is experimentally testable by considering MARs, which naturally contain tunnel processes transferring integer multiples of the elementary charge across the junction.

Tunneling in an SIS junction must be understood in the framework of MARs, the scattering processes permitting the conversion of a normal current into a supercurrent. An electron incident onto the superconductor from within the barrier 


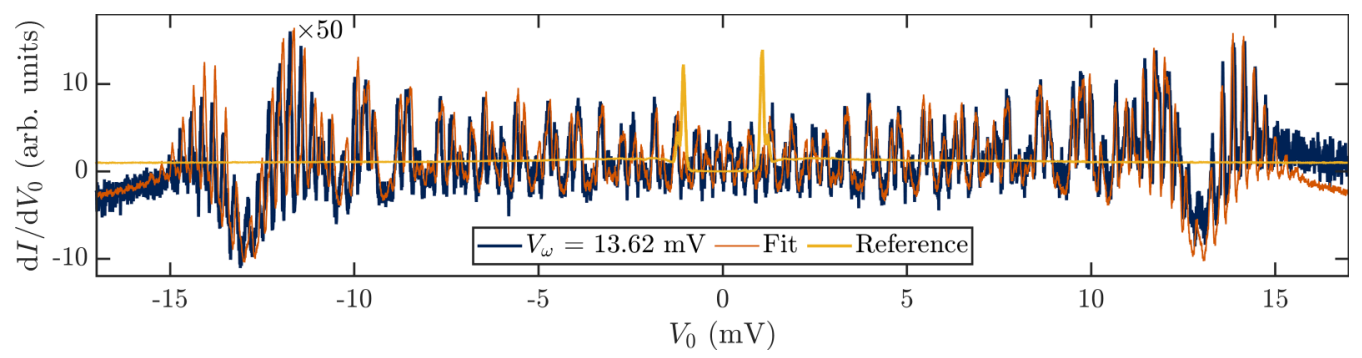

FIG. 3. Junction at high microwave amplitude: Conductance spectrum of an unperturbed SIS junction (yellow) and of the same junction under irradiation by the maximum possible RF signal in our setup (dark blue), equivalent to $V_{\omega}=13.62 \mathrm{meV}$ at $70.02 \mathrm{GHz}$. The orange curve is a fit to the data using Eq. (1) and the reference spectrum in yellow as input data. Even at these extreme conditions, the data is well reproduced. Discrepancies on the high positive bias side are due to a slight and unavoidable $z$ drift during data acquisition. The in-gap peaks in the reference spectrum arise from a magnetic impurity state on the tip. $G_{N}=5 \times 10^{-3} G_{0}$.

is reflected as a hole, thereby transferring a charge of $2 e$ into the superconductor and forming a Cooper pair. As particles of opposite charge are traveling in opposite directions, MAR processes can result in the transfer of multiple elementary charges across the junction. There is thus no quasiparticle analog to Cooper pairs in transport through Andreev reflections to which the microwave signal could be coupled and it is likely that the interactions of all particles participating in an Andreev process need to be considered explicitly to form a theoretical model of the process. This is the approach of the microwave-assisted MAR model in Ref. [19].

To investigate the effect of the microwave signal on Andreev transport, we increase the normal state conductance of the junction by reducing the tip-sample distance. A representative conductance spectrum of a high conductance junction with $\left(G_{N}=1.07 G_{0}\right)$ can be found in Fig. 5(a) in dark blue. In addition to the coherence peak located at $\approx 1.22 \mathrm{mV}$, there are now a series of subgap features related to MARs. Most prominent are the first- and second-order MAR peaks at $\approx 0.7 \mathrm{meV}$ and $\approx 0.36 \mathrm{meV}$, respectively. A spectrum of the same junction under microwave irradiation at $60 \mathrm{GHz}$ is shown in Fig. 5(a) in orange. At first glance, the data seems to support the idea of a charge-sensitive measurement as replica from the first and second MAR are found at the correct offsets of $\approx \frac{\hbar \omega}{2 e}$ and $\approx \frac{\hbar \omega}{3 e}$, respectively.

A quantitative analysis of the data requires a thorough characterization of the junction. This means, ideally, to determine the number of conduction channels and their respective transmission probabilities, which are generically known as the junction PIN code. This can be done by analyzing the subgap structure in the absence of microwaves using the standard MAR theory and fitting procedures that are well described in the literature [26,37-41]. For the case of the data shown in Fig. 5(a), the PIN code analysis finds a total of five transport channels with transmissions $\tau_{i}=[0.416,0.293,0.115,0.114$, 0.112] (see Supplemental Material for more details on the PIN code analysis) [22]. This is consistent with the $d$-band nature of vanadium $[39,40]$. In addition to the junction PIN code, the amplitude of the microwave signal at the junction needs to be known. It is determined by acquiring a conductance spectrum in the tunneling regime, where Eq. (1) can be applied, and performing a fit as described above.

Modeling the data according to the Tien-Gordon theory further requires knowledge of the magnitudes of current contributions carrying $m$ elementary charges. Such a decomposition of the total current is possible through full counting statistics (FCS), where it is found that multiple charge processes make a significant contribution to the total current across the junction and even dominate in the subgap regime at high conductance [42-44]. The separate current contributions can then be treated using an adapted version of Eq. (1) and the results compared to the MAR calculation [19].

The result of the FCS calculation as well as the individual contributions to the total conductance are shown in Fig. 5(b). The discrepancy around the first MAR and additional structure below approximately $0.5 \mathrm{mV}$ bias can be attributed to the difficulty in correctly accounting for broadening effects at the ultra-low temperature of the experiment [36,45]. Applying Eq. (1) to the FCS result, accounting for multiple charge transfers in the individual contributions, yields the purple curve in Fig. 5(c) (TG ansatz). The total conductance obtained deviates strongly from the experimental data, shown in orange, even qualitatively. This approach is not able to correctly reproduce the location of the peaks in the experimental data, nor their magnitude. The microwave-assisted MAR model, shown in cyan in Fig. 5(c), gives a much better description of the data. In particular, the location of the satellite peaks of the first MAR are predicted accurately. The error in their height can be traced by to the original issue overestimating the magnitude of the first MAR in the FCS model [22].

\section{CONCLUSIONS}

The essential difference between the Tien-Gordon and microwave-assisted MAR models lies in the interaction between $\mathrm{dc}$ transport and the microwave signal. The TienGordon ansatz assumes a clear separation. This view of tunneling breaks down when higher-order processes are included. The exact calculation, on the other hand, sees dc transport and microwave effects as linked at the most fundamental of levels. Quasiparticles interact with the microwave signal as part of the tunneling process, absorbing or emitting packets of energy. For the case of higher-order processes such as MARs, a pair of initial and final states may be linked through several pathways involving different interactions with the microwave field. Transport in the presence of an ac driving signal must then be understood as a sum over histories of particles traveling back and forth across the barrier. The success of the MAR model can thus be seen as a direct consequence of multiple 

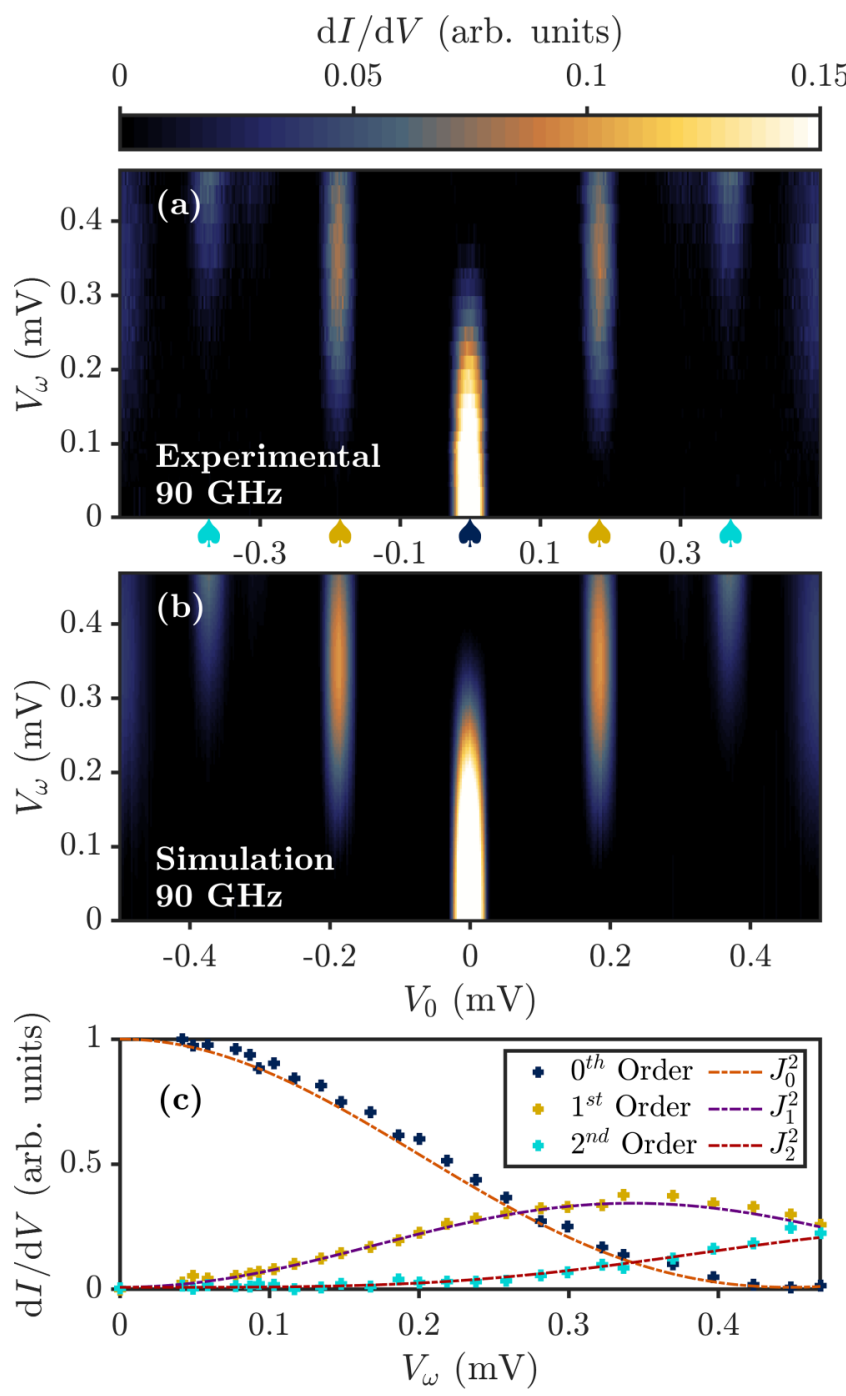

FIG. 4. The Josephson effect under microwave irradiation: (a) Stacked contour plot of a series of conductance spectra of an SIS junction under irradiation by a $90 \mathrm{GHz}$ signal at varying $V_{\omega}$ with a focus on the Josephson effect. Satellite peaks now appear at an offset of $\hbar \omega / 2$. Markers on the $x$ axis indicate the locations of the line profiles presented in (c). $G_{N}=7.6 \times 10^{-3} G_{0}$. (b) Simulation of the data in panel a) using an experimental conductance spectrum and Eq. (2). (c) Constant $V_{0}$ profiles of the experimental data in (a) at the principal Jospehson peak $\left(V_{0}=0 \mathrm{mV}\right.$, dark blue) and the first $\left(V_{0}= \pm 0.185 \mathrm{mV}\right.$, yellow) and second $\left(V_{0}= \pm 0.372 \mathrm{mV}\right.$, cyan) satellites, normalized to the maximum of the Josephson peak at $V_{\omega}=0$. The dashed-dotted lines are the Bessel functions of zeroth (orange), first (purple), and second (red) order in $\frac{2 e V_{\omega}}{\hbar \omega}$.

reflections occurring within the barrier and the interference of the particles involved.

An ac driving signal applied to a tunnel junction cannot be seen as only exciting fundamental modes within the sample, or as a simple broadening mechanism. The conductance of the junction will necessarily be modified. In the simplest cases, such as normal conducting samples and SIN or SIS junctions well below $0.1 G_{0}$, this modification is well captured by the Tien-Gorden Eq. (1) and is equivalent to a redistribution of the density of states of the electrodes. This simple model

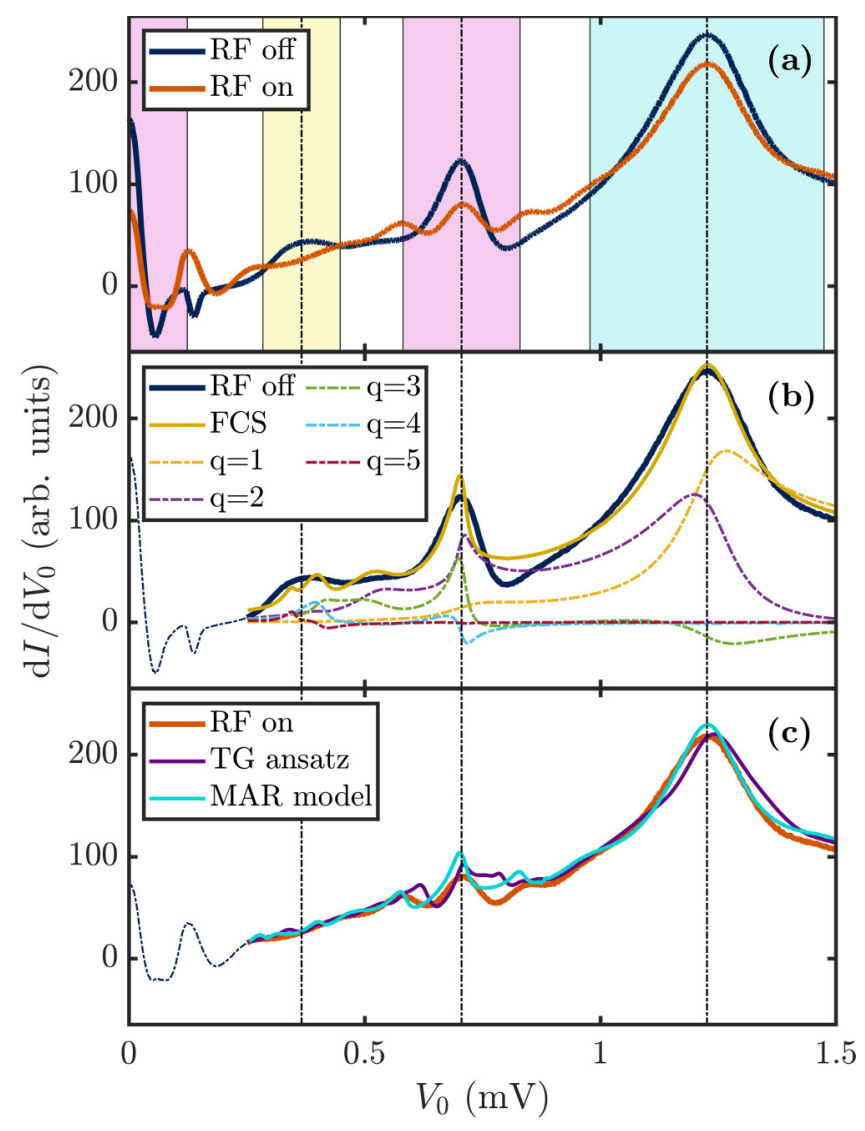

FIG. 5. Modeling the interaction with the microwave signal: (a) Conductance spectrum at $G_{N}=1.07 G_{0}$ in absence (dark blue) and presence (orange) of a microwave signal. The shaded areas mark energy offsets of $\hbar \omega$ (cyan), $\hbar \omega / 2$ (purple) and $\hbar \omega / 3$ (yellow). (b) Experimental conductance spectrum (dark blue) and FCS model thereof (yellow). The individual contributions to the conductance from currents carrying $q=1,2,3,4,5$ charges are shown in yellow, purple, green, cyan, and red, respectively. The Josephson effect is not included in the model. (c) Comparison of the TG ansatz (purple) and exact calculation (cyan) to the experimental data (orange). The MAR theory gives a significantly better description of the data than the TG ansatz.

breaks down when higher-order effects become significant. In superconducting junctions in particular, this is the case as soon as the first Andreev reflection contributes significantly to the current. More elaborate models are needed to understand the junction behavior in these cases [19].

Microwave-assisted tunneling is a significant expansion of the STM toolbox. By pulling back the curtain over higherorder tunnel processes, a microwave signal may be used to reveal fine details about the nature of quantum mechanical transport, which are inherently unavailable in a pure dc measurement. In the case of SIS junctions in particular, the interference between the microwave signal and the inherent ac components of the supercurrent may be a pathway to observing signatures of the ac Josephson effect in superconducting point contacts.

Note added. Recently, we became aware of a related work [46]. 


\section{ACKNOWLEDGMENTS}

The authors would like to thank Alfredo Levy-Yeyati, Ciprian Padurariu, Björn Kubala, and Manish Garg for insightful discussions. J.C.C. acknowledges funding from the Spanish Ministry of Economy and Competitiveness
(MINECO) (Contract No. FIS2017-84057-P). J.A. acknowledges support from the IQST and the German Science Foundation (DFG) under Grant No. AN336/11-1. This work was funded in part by the ERC Consolidator Grant AbsoluteSpin (Grant No. 681164).
[1] G. Binnig, H. Rohrer, C. Gerber, and E. Weibel, Phys. Rev. Lett. 49, 57 (1982).

[2] S. Loth, M. Etzkorn, C. P. Lutz, D. Eigler, and A. J. Heinrich, Science 329, 1628 (2010).

[3] Y. Terada, S. Yoshida, O. Takeuchi, and H. Shigekawa, J. Phys.: Condens. Matter 22, 264008 (2010).

[4] T. L. Cocker, V. Jelic, M. Gupta, S. J. Molesky, J. A. J. Burgess, G. De Los Reyes, L. V. Titova, Y. Y. Tsui, M. R. Freeman, and F. A. Hegmann, Nature Photon. 7, 620 (2013).

[5] S. Yoshida, Y. Aizawa, Z.-h. Wang, R. Oshima, Y. Mera, E. Matsuyama, H. Oigawa, O. Takeuchi, and H. Shigekawa, Nature Nanotechnol. 9, 588 (2014).

[6] S. Baumann, W. Paul, T. Choi, C. P. Lutz, A. Ardavan, and A. J. Heinrich, Science 350, 417 (2015).

[7] M. Rashidi, J. A. Burgess, M. Taucer, R. Achal, J. L. Pitters, S. Loth, and R. A. Wolkow, Nature Commun. 7, 13258 (2016).

[8] T. L. Cocker, D. Peller, P. Yu, J. Repp, and R. Huber, Nature (London) 539, 263 (2016).

[9] W. Paul, K. Yang, S. Baumann, N. Romming, T. Choi, C. P. Lutz, and A. J. Heinrich, Nature Phys. 13, 403 (2017).

[10] K. Yang, P. Willke, Y. Bae, A. Ferrón, J. L. Lado, A. Ardavan, J. Fernández-Rossier, A. J. Heinrich, and C. P. Lutz, Nature Nanotechnol. 13, 1120 (2018).

[11] M. Garg and K. Kern, Science 367, 411 (2020).

[12] P. Willke, K. Yang, Y. Bae, A. J. Heinrich, and C. P. Lutz, Nature Phys. 15, 1005 (2019).

[13] K. Yang, W. Paul, S.-H. Phark, P. Willke, Y. Bae, T. Choi, T. Esat, A. Ardavan, A. J. Heinrich, and C. P. Lutz, Science 366, 509 (2019).

[14] S. Shapiro, Phys. Rev. Lett. 11, 80 (1963).

[15] P. Tien and J. Gordon, Phys. Rev. 129, 647 (1963).

[16] G. Falci, V. Bubanja, and G. Schön, Z. Phys. B 85, 451 (1991).

[17] M. Chauvin, P. Vom Stein, H. Pothier, P. Joyez, M. E. Huber, D. Esteve, and C. Urbina, Phys. Rev. Lett. 97, 067006 (2006).

[18] A. Roychowdhury, M. Dreyer, J. R. Anderson, C. J. Lobb, and F. C. Wellstood, Phys. Rev. Appl. 4, 034011 (2015).

[19] J. C. Cuevas, J. Heurich, A. Martín Rodero, A. Levy Yeyati, and G. Schön, Phys. Rev. Lett. 88, 157001 (2002).

[20] P. W. Davies and R. M. Lambert, Surf. Sci. 95, 571 (1980).

[21] J. S. Foord, A. P. C. Reed, and R. M. Lambert, Surf. Sci. 129, 79 (1983).

[22] See Supplemental Material at http://link.aps.org/supplemental/ 10.1103/PhysRevB.101.134507 for further details on data modelling, heating effects of the microwave radiation, the classical nature of field driven tunnelling, and sequential charge tunnelling.

[23] M. I. Salkola, A. V. Balatsky, and J. R. Schrieffer, Phys. Rev. B 55, 12648 (1997).
[24] H. Huang, R. Drost, J. Senkpiel, C. Padurariu, B. Kubala, A. L. Yeyati, J. C. Cuevas, J. Ankerhold, K. Kern, and C. R. Ast, arXiv:1912.05607.

[25] E. N. Bratus, V. S. Shumeiko, and G. Wendin, Phy. Rev. Lett. 74, 2110 (1995).

[26] J. C. Cuevas, A. Martín-Rodero, and A. Levy Yeyati, Phys. Rev. B 54, 7366 (1996).

[27] O. H. Soerensen, B. Kofoed, N. F. Pedersen, and S. Shapiro, Phys. Rev. B 9, 3746 (1974).

[28] L. P. Kouwenhoven, S. Jauhar, K. McCormick, D. Dixon, P. L. McEuen, Y. V. Nazarov, N. C. van der Vaart, and C. T. Foxon, Phys. Rev. B 50, 2019 (1994).

[29] S. E. de Graaf, J. Leppäkangas, A. Adamyan, A. V. Danilov, T. Lindström, M. Fogelström, T. Bauch, G. Johansson, and S. E. Kubatkin, Phys. Rev. Lett. 111, 137002 (2013).

[30] T. Kampfrath, K. Tanaka, and K. A. Nelson, Nature Photon. 7, 680 (2013).

[31] M. Tinkham, Introduction to Superconductivity, 2nd ed. (Dover Publications, Mineola, 1996).

[32] H. Grabert, Phys. Rev. B 92, 245433 (2015).

[33] D. V. Averin, Y. Nazarov, and A. A. Odintsov, Physica B 165, 945 (1990).

[34] M. H. Devoret, D. Esteve, H. Grabert, G. L. Ingold, H. Pothier, and C. Urbina, Phys. Rev. Lett. 64, 1824 (1990).

[35] G. L. Ingold, H. Grabert, and U. Eberhardt, Phys. Rev. B 50, 395 (1994).

[36] C. R. Ast, B. Jäck, J. Senkpiel, M. Eltschka, M. Etzkorn, J. Ankerhold, and K. Kern, Nature Commun. 7, 13009 (2016).

[37] D. Averin and A. Bardas, Phys. Rev. Lett. 75, 1831 (1995).

[38] E. Scheer, P. Joyez, D. Esteve, C. Urbina, and M. H. Devoret, Phys. Rev. Lett. 78, 3535 (1997).

[39] E. Scheer, N. Agraït, J. C. Cuevas, A. Levy Yeyati, B. Ludoph, A. Martín Rodero, G. R. Bollinger, J. M. van Ruitenbeek, and C. Urbina, Nature (London) 394, 154 (1998).

[40] J. C. Cuevas, A. Levy Yeyati, and A. Martín-Rodero, Phys. Rev. Lett. 80, 1066 (1998).

[41] J. Senkpiel, S. Dambach, M. Etzkorn, R. Drost, C. Padurariu, B. Kubala, W. Belzig, A. Levy Yeyati, J. C. Cuevas, J. Ankerhold, C. R. Ast, and K. Kern, arXiv:1810.10609v1.

[42] J. C. Cuevas and W. Belzig, Phys. Rev. Lett. 91, 187001 (2003).

[43] G. Johansson, P. Samuelsson, and Å. Ingerman, Phys. Rev. Lett. 91, 187002 (2003).

[44] J. C. Cuevas and W. Belzig, Phys. Rev. B 70, 214512 (2004).

[45] B. Jäck, M. Eltschka, M. Assig, M. Etzkorn, C. R. Ast, and K. Kern, Phys. Rev. B 93, 020504(R) (2016).

[46] O. Peters, N. Bogdanoff, S. A. Gonzalez, L. Melischek, J. R. Simon, G. Reecht, C. B. Winkelmann, F. von Oppen, and K. J. Franke, arXiv:2001.09534. 